MS092.001

\title{
Microsymposium
}

\section{Chitosan-engineered metal-organic frameworks as oral drug nanocarriers}

Patricia Horcajada ${ }^{1}$, Tania Hidalgo ${ }^{2}$, Monica Gimenez-Marques ${ }^{2}$, Rosana Simon-Vazquez ${ }^{3}$, Jose Avila ${ }^{4}$, Maria C Asensio ${ }^{5}$, Fabrice Salles $^{6}$, Christian Serre ${ }^{2}$, Africa Gonzalez-Fernandez ${ }^{3}$

${ }^{1}$ IMDEA Energy, Mostoles-madrid, Spain, ${ }^{2}$ Institut Lavoisier, CNRS UMR 8180, Versailles, France, ${ }^{3}$ Immunology, Biomedical Research Center (CINBIO) and Institute of Biomedical Research of Vigo (IBIV), Universidad de Vigo, Vigo, Spain, ${ }^{4}$ Synchrotron SOLEIL \& Université Paris-Saclay, Gif-sur-Yvette Cedex, France, ${ }^{5}$ Synchrotron SOLEIL \& Université Paris-Saclay, Gif Sur Yvette, France,

${ }^{6}$ ICGM - UMR5253- Equipe AIME, Université Montpellier II, Montpellier, France

E-mail: patricia.horcajada@imdea.org

The involvement of Metal-Organic Frameworks (MOFs) in biomedical applications is currently one of the hot topics in the emerging field of hybrid porous solids.[1] In particular, nanometric MOFs (nanMOFs) have recently attracted a great deal of attention owing to their large porosity and versatile composition, enabling to entrap remarkable loadings of a wide variety of challenging active molecules and progressive releases, together with imaging properties.[2] However, prior to any practical use of nanoMOFs, it is of a high societal relevance to investigate their biostability, biocompatibility and biodistribution as a function of the administration route. Up to now, most studies have targeted the intravenous route, related to pain and severe complications; whereas nanoMOFs for oral administration, a commonly used non-invasive and simpler route, remains however unexplored.

We propose here the biofriendly preparation of a suitable oral nanocarrier based on the benchmarked biocompatible mesoporous iron(III) trimesate MIL-100(Fe) nanoparticles coated with the bioadhesive polysaccharide chitosan (CS). The resulting CS-engineered MIL-100(Fe) nanoparticles were fully characterized, with particular attention to their structural, chemical and colloidal stability under physiological oral conditions. A combination of high-resolution X-ray Absorption NearEdge Structure (XANES) and computing simulation approaches allowed understanding the interactions between the CS and the nanoparticles surface. Finally, the ability to bypass the intestinal barrier of these nanoparticles was also investigated under in vitro conditions.[3]

[1] Horcajada P. et al. (2012) Chem.Rev., 112, 1232-1268

[2] Gimenez-Marques M. et al. (2016) Coord. Chem. Rev., 307, 342-360

[3] Hidalgo T. et al. (2017) Sci. Rep., DOI:10.1038/srep43099

Keywords: MOFs, drug nanocarriers, oral administration 